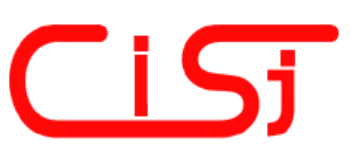

\title{
SOM BASED DECISION SUPPORT IN FAILURE MANAGEMENT
}

\author{
Miki Sirola, Golan Lampi, Jukka Parviainen
}

\author{
Helsinki University of Technology \\ Laboratory of Computer and Information Science \\ P.O.Box 5400, FIN-02015 HUT, Finland \\ Miki.Sirola@hut.fi, Golan.Lampi@hut.fi, Jukka.K.Parviainen@hut.fi
}

\begin{abstract}
Computerized decision support system field covers many methodologies and application areas. In this paper Self-Organizing Map (SOM) and knowledge-based techniques are used in combination to reason problematic situations in failure management. A process model that consists of individual connected process components has been developed. A primary circuit of a boiling water nuclear power plant including two branches has been composed. A failure management scenario is thoroughly analyzed and solved with the SOM based decision support system. The structure and reasoning of the Computerized Decision Support System (CDSS) is also shortly discussed. The process model is demonstrated together with the CDSS and shown to be useful. The tool helps operators decision making with various visualizations, and by giving concrete recommendations for possible control actions or other acts.
\end{abstract}

Keywords: Self-Organizing Map, Decision Support, Failure Management

\section{INTRODUCTION}

Computerized decision support systems have developed to their current status during many decades. Various methodologies and application areas have been introduced during this time. One important milestone was reached when knowledgebased techniques were presented first time in 1980s. A complete description of decision support field is written in [1] and [2].

Self-Organizing Map (SOM) [3] is an artificial neural networks algorithm based on competitive learning. SOM is effective in analysis and visualisation of multi-dimensional data. It helps in mapping nonlinear statistical dependencies from multi-dimensional measurement data into simple geometrical relations, mostly in two-dimensional space. The map maintains roughly the most important topological and metric relations from the original measurement element, and clusters the data. Clustering is needed for instance in data analysis of complex processes and systems. The SOM has been used in numerous engineering applications [4].

The main problems in failure management are often in detection and identification of the possible faults. A knowledge-based approach has been used in [5] to solve these problems, and a computerized decision support system prototype for fault diagnosis of safety critical processes has been built.

The role of the Decision Support System (DSS) in a process monitoring task is presented in Figure 1. Measurement data from the process is used either directly or after preprocessing. The output of the decision support system is presented to the operator with a Man-Machine Interface (MMI) system. Process is never controlled directly by a decision support system, but the operator can use this information or possible advice when deciding control actions from several possibilities.

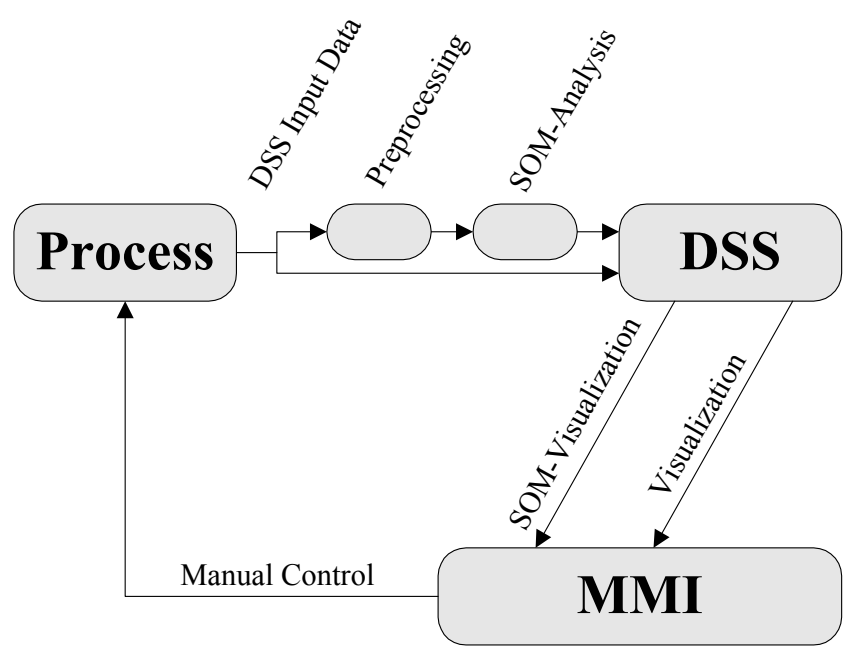

Fig.1. The role of DSS assisted by SOM analysis in a process control problem.

Based on a compatibility analysis [6] a prototype of a computerized decision support system using SOM method (DERSI) has been built [7]. In the first 
phase artificial data and then data from a simple error state model [8] built in Simulink was used. In this paper we present a process model producing data for DERSI, and a failure management case example from an imaginary nuclear power plant. The prototype structure and reasoning are also shortly introduced.

We are able to show the usefulness of SOM method in a computerized decision support system by analyzing data got from the process model. Normal process data and failure data are compared in a scenario from the nuclear industry. Neural methods and knowledge-based methods complement each other, and together a powerful tool for failure management is accomplished.

\section{PROTOTYPE STRUCTURE AND REASONING}

The DERSI prototype is described in detail in [7]. It is a Matlab software program built on top of Matlab extension SOMToolbox [9], which was developed earlier in the Laboratory of Computer and
Information Science in Helsinki University of Technology. DERSI object structure is presented in Figure 2.

The rule base of DERSI is under development. A small rule base for testing and demonstration purposes already exists. It is based on ordinary IF THEN rules that have an associated priority value. The current version of the prototype is based on rule-based and case-based reasoning. Some rules recommend actions if certain predicates are true, e.g. pressure is dangerously high. Other rules offer a diagnosis, but do not recommend an action. These rules are based on SOM quantisation error.

Multiple rules can be fired simultaneously. If multiple rules are fired, they are shown to the operator in the priority order. Action rules and diagnostics rules are in the same recommendation list. The rules are in a mapping matrix of a DecisionDatabase class of DERSI. The mapping matrix has sensor columns, predicate columns, decision recommendation columns and a priority column.

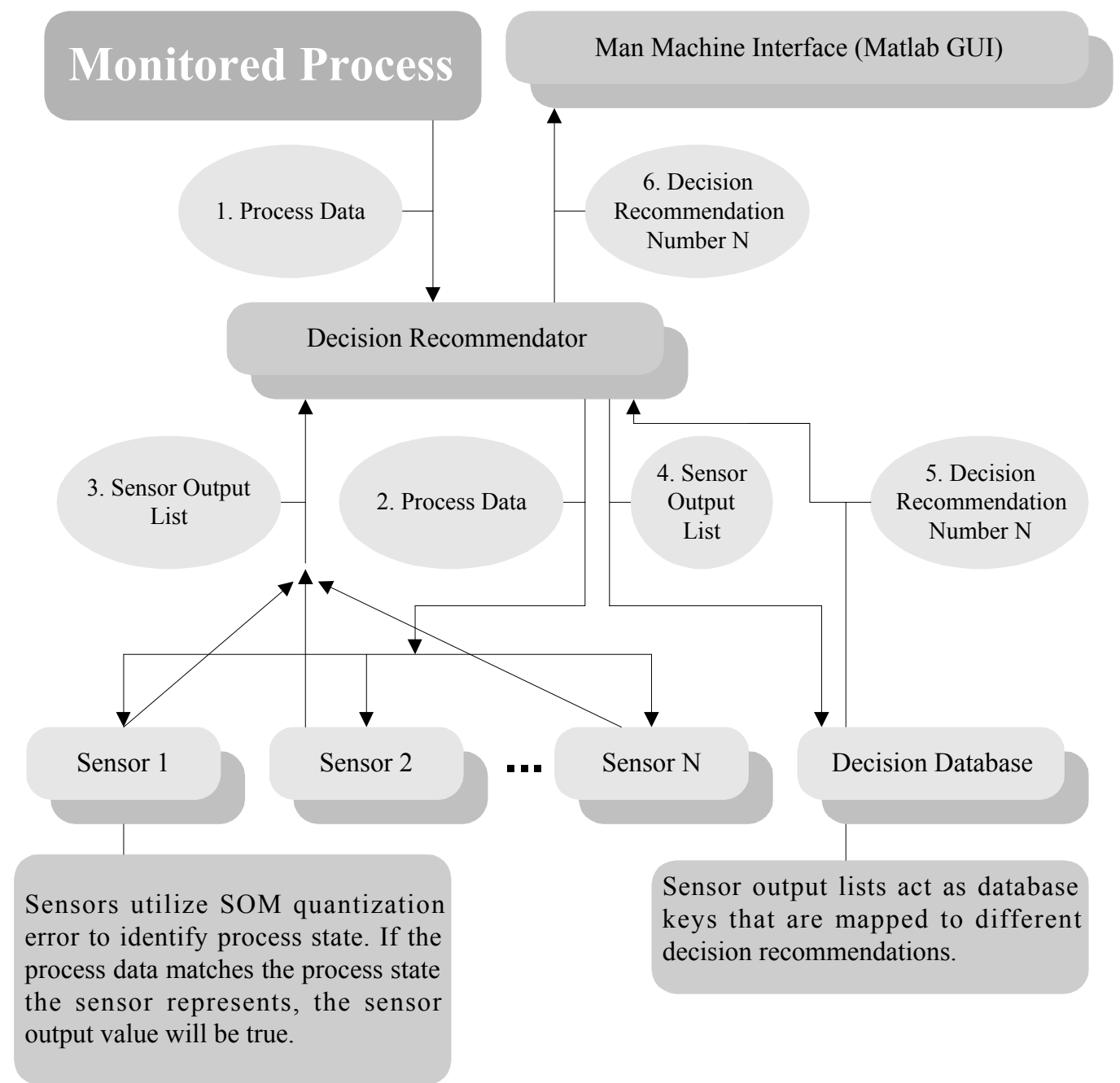

Fig. 2. DERSI object structure. A semiformal presentation of the most important parts and data flows in DERSI. The data flows are numbered in chronological order. 
Current version of DERSI does not support hierarchical rule base. It does not yet implement true inference such as backward chaining or forward chaining, but it has mappings equivalent to rules. A hierarchical rule base needs to be flattened to make it possible to translate it to DERSI mappings. Then every path from a decision tree root to a decision tree leaf corresponds to a DERSI mapping.

Currently the model is polling the sensor values regularly. There is no special stimulus to trigger the inference process. The decision recommendations are in order of importance.

\section{PROCESS MODEL}

A process model has been built to produce data for the SOM based decision support system (DERSI). The process model is realized in Matlab Simulink environment. Developing the process components has begun from equations based on physical laws, but the correct behaviour has been realized by experimentally tuning various parameters of the model. The process components have been realized for pump, valve, preheater, turbine, condenser, reactor, pipe, branch, etc.; process components used on a nuclear power plant. By combining these process components a simplified primary circuit of a boiling water nuclear power plant including two branches has been constructed, see Figure 3. Also the cooling system of both branches in the primary circuit is included in the model.

The water and steam circulation has been realized in two phases, which makes the model rather complex. In the first version of the model instability and some other incorrect behaviour is met with in some situations. In most simulated examples the physical quantities behave mostly correctly. The model is already good enough for testing purposes and big help in the further development of DERSI. In addition to normal operation numerous fault situations have been simulated, and one of these scenarios is presented in this paper.

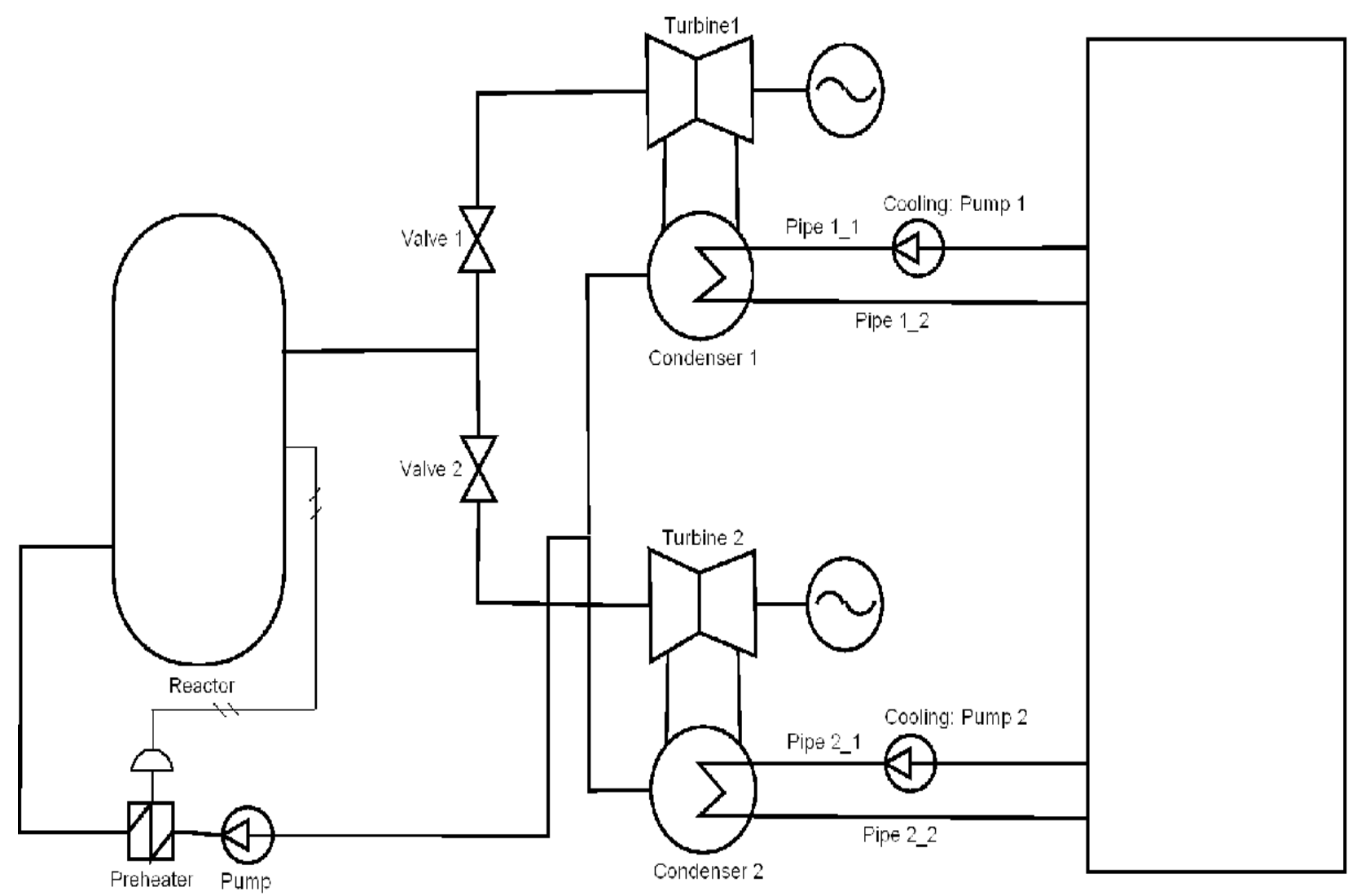

Fig. 3. PI-diagram of the Simulink process model.

The detailed process component models including about 80 equations, and detailed realizations in Simulink environment including detailed diagrams can be found from [10]. The modelling begins with an equation of a flow in a cylindrical pipe. Behaviour of flow, pressure and temperature, etc. basic variables are important base elements in the model. The model components need to be interconnected. The connection points of the various components are divided into inputs and outputs. They are in an important role to make the whole system act in a sensible way.

Because simulated process components never brake as the real components sometimes do, such 
disturbances need to be simulated separately. For instance, a leak can be simulated by adding an extra valve to the model. Therefore, some extra components have been derived to be able to simulate different disturbances and failures.

The current version of the model includes several simulated demonstration scenarios. One of them, a malfunction in an actuator in one turbine branch, is described in detail in the next section. Demonstration scenarios for a leakage in a reactor input pipe in the primary circuit, a leakage between one turbine and condenser, a leakage in the secondary cooling system, and a disturbance in the input power also exist.

\section{FAILURE MANAGEMENT SCENARIO}

The failure management scenario is described as follows. For one reason or another, the admission valve in the second branch of the primary circuit (Valve 2 in Figure 3) suddenly closes when it should stay open to let the steam flow into the turbine. The pressure before the valve begins to increase and pressure after the valve to decrease. The curves of most important quantities are seen in Figure 4 and the DERSI MMI (Man-Machine Interface) in this scenario in Figure 5.

Quantisation error is one important method in the DERSI reasoning process as it has been explained in the previous papers about this topic [7]. In this scenario the rules are fired when certain threshold values are reached for certain quantities, such as pressure in the following branch after reactor (in the direction of mass flow), turbine pressure, or already earlier mentioned quantisation error.

The first recommended action is to try to reopen the admission valve in the second branch. Then, if this is not possible, the next recommended action is to open the bypass valve. Then the steam flow would go directly to the condenser bypassing the turbine, and the pressures on both sides would return closer to normal values. Note that the recommendations in Figure 5 are in the priority order rather late in the scenario development, and not anymore in the recommendation order.

If there would be a malfunction also in the bypass valve, then the next recommendation is to open the relief valve and also to initiate the Auxiliary Feedwater System (AFS). In this case the high pressure steam would be released directly to the containment, and also the possibility of a radioactive release would increase. The AFS system is needed to replace the missing mass (water or steam) in the primary circuit.

In case of a malfunction in the relief valve (or even without it) the next recommendation is to prepare for a possible reactor shutdown. Note that the bypass valve and relief valve are not modelled in the Simulink process model. In the DERSI MMI in Figure 5 it is also seen the effects and costs of each recommended action. The desired effects are to return the process back into a stable state. The costs are low in the two latter options with lower priorities and high in the two first options with higher priority. The diagnosis result is not seen on the screen at the moment, because there is no space left in the recommendation block, and therefore it has rolled out of the screen.

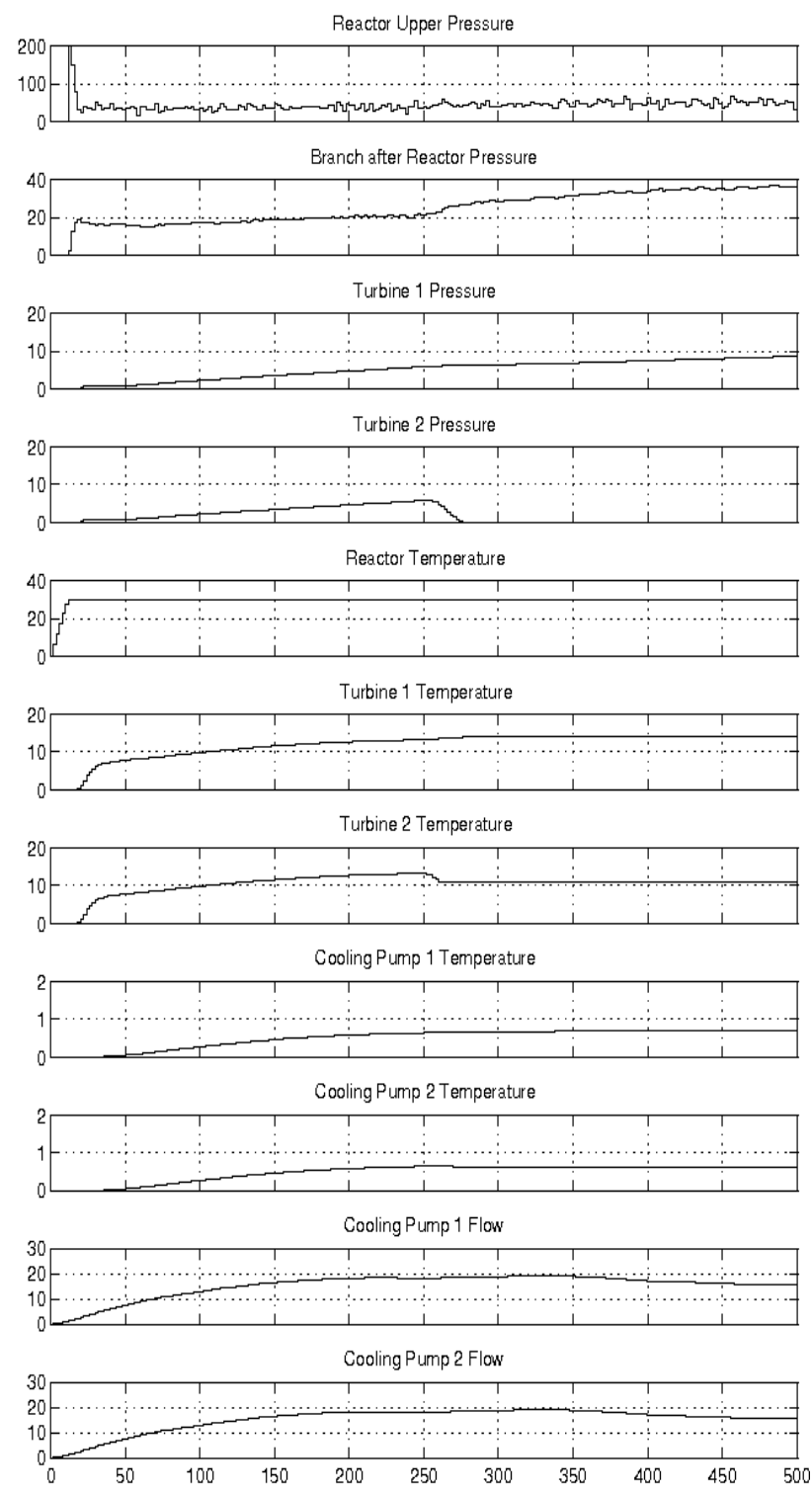

Fig. 4. Data of the fault state in the scenario. The failure occurs at time point 250 .

In addition to the decision recommendations in the six fields of DERSI MMI some operating menus of the tool, U-matrix (a visual tool calculating distances that reveals the cluster structure), time series curves and SOM mappings of 11 selected variables can be found. The white trajectory in the state U-matrix moves from the middle area into the 
left corner when the failure occurs. (The fault areas of other demonstration scenarios in U-matrix are also marked in the same MMI field). The SOM maps are twice on the screen to make it possible to compare the maps of normal operation (the lower maps) and failure (upper maps). For instance, in the pressures involved in this failure clear differences can be noticed in corresponding maps.

In the data of normal operation the pressures in both turbines correlate, as well as the pressure directly after reactor. This correlation between pressures in the turbines has turned into reverse correlation in the failure data. This can be read from the reverse colouring of the corresponding SOM maps. In the pressure of Turbine 2 the dark corner has changed into almost white (in the black and white colouring) and vice versa. Smaller changes in the colouring of other SOM maps of other selected variables can also be noticed. The correlation between pressure in not disturbed turbine and pressure just after reactor somewhat remains also in the failure.

In previous papers [7] we have presented a scenario where a leak appears into the primary circuit. Also there significant differences in quantisation error (calculated as a cumulated difference between normal data and failure data) and in visual SOM mappings were noted. A broken pipe is there the cause of the initial problem. In this scenario an actuator malfunction is the initial cause of the problem. In both cases the data can reveal clearly the possible causes of these problems with help of this tool. Most of the DERSI MMI is planned to be a visual tool to help the operator in his decision making. The rule base is used only in producing the recommended actions for the operator. The case based rule base is not explained here in detail.

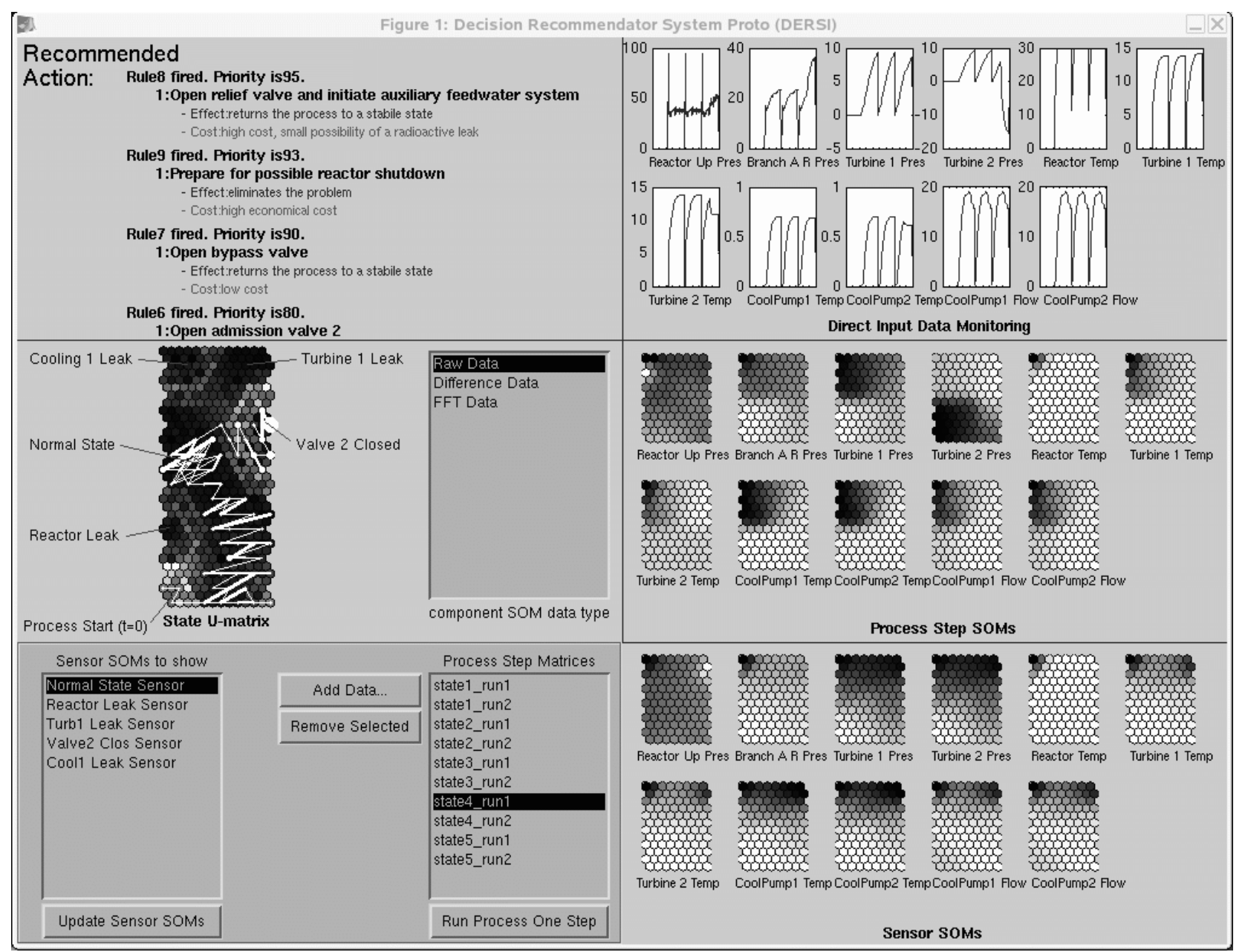

Fig.5. DERSI MMI in the failure scenario.

\section{DISCUSSION}

A SOM based decision support system (DERSI) for failure management has been developed. A process model to produce data for DERSI has been included into the system. The model consists of independent process components, which can be connected to each other via their inputs and outputs. A simplified primary circuit of a boiling water reactor nuclear power plant including two branches has been composed, and a failure management 
scenario has been presented.

We have been able to show how the data produced by the process model is utilized in the decision support tasks, and how it helps also in further development of the system. We can also demonstrate how neural method Self-Organizing Map (SOM) together with knowledge-based methodology helps the decision making process in a computerized decision support system. The reasoning process utilized e.g. quantisation error in concluding the recommended actions in various failures. In the presented scenario we can see in practice an example of a successful reasoning process. In addition the operator is provided with direct visual help by the tool.

DERSI is a general decision support system framework that could be used also in analysis of other domains in addition to industrial processes. The selection of data variables taught to DERSI is one critical point. DERSI is especially useful when there are very many input variables, and it is almost impossible to comprehend from variable plots or even SOM component planes what is really going on in the process.

In the process model examples mostly no measurement noise was added to the data, and no preprocessing was needed either. The quantisation error results were mostly satisfactory. In the Umatrix in most of the states after failures the trajectory began to go to an area that was unique to each state. So the distance between state areas correlated with the distance between fault origins. In fact, this is only true when the distances between fault origins are relatively low.

The state identification problem is difficult. One of the purposes of the process model was to test the recommendations of the DSS, and see if they are able to return the process back to safe state. DERSI decides now discretely the recommendations, and it makes some prioritization.

As it was demonstrated in the scenario, numerical values are given to each recommendation to define their priority order. The prioritization principles are based on expert knowledge as the knowledge itself in the rule base. Later on more advanced methods could be tried out here.

Our future plan is to analyze also real data with DERSI and data from large scale simulators. We have initialized some cooperation with nuclear authorities and power companies to enable further development of DERSI with closer cooperation with the possible end-users. Paper industry could be another alternative where such a system could be used with successful results.

\section{REFERENCES}

[1]. Turban E., Aronson J. Decision support systems and intelligent systems. Prentice Hall 1998.

[2]. Marakas G. Decision support systems in the 21st century. Prentice Hall 1999.

[3]. Kohonen T. The self-organizing map. Springler, Berlin, Heidelberg, 1995.

[4]. Simula O., et.al. The self-organizing map in industry analysis. Industrial applications of neural networks. CRC Press, 1999.

[5]. Sirola M. Computerized decision support systems in failure and maintenance management of safety critical processes. VTT Publications 397. Espoo, Finldand, 1999.

[6]. Sirola M., Vesanto J. Utilization of neural methods in knowledge-based decision support systems - state monitoring as a case example. IASTED International Conference for Modelling, Identification and Control. Innsbruck, Austria, 2000.

[7]. Sirola M. et.al. Neuro computing in knowledgebased decision support systems. EHPG-Meeting of OECD Halden Reactor Project. Sandefjord, Norway, 2004.

[8]. Sirola M. et.al. Using self-organizing map in a computerized decision support system. International Conference on Neural Information Processing (ICONIP'2004). Calcutta, India, 2004.

[9]. Vesanto J., et.al. Technical report on SOM Toolbox 2.0. Espoo, Finland, 2000.

[10]. Lampi G. Building a process model to produce data for the computerized decision support system using self-organizing map. Technical report. Espoo, Finland, 2004.

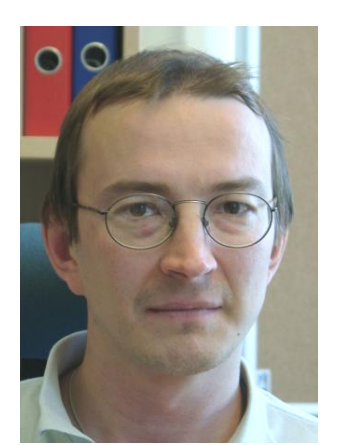

Miki Sirola has been a Laboratory Engineer in the Laboratory of Computer and Information Science in Helsinki University of Technology since 1998. He received MSC 1988 in Electrical Engineering (System Control and Automation) from Helsinki University of Technology, LicTech 1993 in Electrical Engineering (Automation) and DTech 1999 in Automation and System Technology (Automation) from the same university. Prior to Helsinki University of Technology, he worked at VTT (Technical Research Centre of Finland) Automation as research scientist (19871998) and at Institutt for Energiteknikk (OECD Halden Reactor Project) as research scientist (19921993). He was nominated a Docent in Computerized Decision Support in 2005 in the Department of 
Computer Science and Engineering in Helsinki University of Technology.

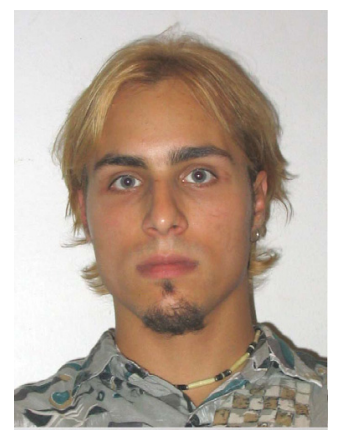

Golan Lampi has been a Research Assistant in the Laboratory of Computer and Information Science in Helsinki University of Technology since 2003. He is a student in the Master of Science program. He has been working in a project where neural methods and knowledge-based methods have been combined in a computerized decision support framework.

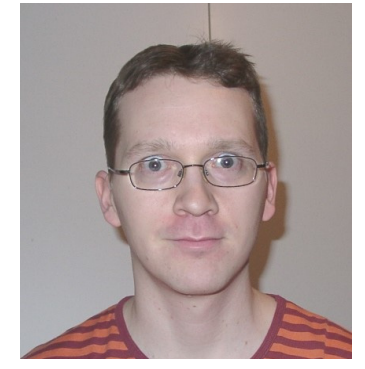

Jukka Parviainen is an Assistant in the Laboratory of Computer and Information Science in Helsinki University of Technology. He received his MSc 2002 in Computer Science from Helsinki University of Technology. His research interests are digital signal processing and data analysis, especially using Self-Organizing Map (SOM). 